\title{
BMJ Open The assessment and impact of sarcopenia in lung cancer: a systematic literature review
}

\author{
Jemima Collins, ${ }^{1}$ Simon Noble, ${ }^{2}$ John Chester, ${ }^{3}$ Bernadette Coles, ${ }^{4}$ Anthony Byrne ${ }^{5}$
}

To cite: Collins J, Noble S, Chester J, et al. The assessment and impact of sarcopenia in lung cancer: a systematic literature review. BMJ Open 2014;4:e003697. doi:10.1136/bmjopen-2013003697

- Prepublication history for this paper is available online. To view these files please visit the journal online (http://dx.doi.org/10.1136/ bmjopen-2013-003697).

Received 1 August 2013 Revised 20 November 2013 Accepted 2 December 2013

CrossMark

\footnotetext{
${ }^{1}$ Cardiff University, Cardiff, UK

2Department of Palliative Medicine, Cardiff University, Cardiff, South Wales, UK ${ }^{3}$ Department of Medical Oncology, Cardiff University, Cardiff, South Wales, UK ${ }^{4}$ Cancer Research Wales Library, Velindre NHS Trust, Cardiff, South Wales, UK ${ }^{5}$ Marie Curie Palliative Care Research Centre, Cardiff University, Cardiff,

South Wales, UK
}

Correspondence to Dr Jemima Collins; jemimacollins@doctors.net.uk

\begin{abstract}
Objectives: There is growing awareness of the relationship between sarcopenia (loss of muscle mass and function), and outcomes in cancer, making it a potential target for future therapies. In order to inform future research and practice, we undertook a systematic review of factors associated with loss of muscle mass, and the relationship between muscle function and muscle mass in lung cancer, a common condition associated with poor outcomes.
\end{abstract}

Design: We conducted a computerised systematic literature search on five databases. Studies were included if they explored muscle mass as an outcome measure in patients with lung cancer, and were published in English.

Setting: Secondary care.

Participants: Patients with lung cancer.

Primary outcome: Factors associated with loss of muscle mass and muscle function, or sarcopenia, and the clinical impact thereof in patients with lung cancer. Results: We reviewed 5726 citations, and 35 articles were selected for analysis. Sarcopenia, as defined by reduced muscle mass alone, was found to be very prevalent in patients with lung cancer, regardless of body mass index, and where present was associated with poorer functional status and overall survival. There were diverse studies exploring molecular and metabolic factors in the development of loss of muscle mass; however, the precise mechanisms that contribute to sarcopenia and cachexia remain uncertain. The effect of nutritional supplements and ATP infusions on muscle mass showed conflicting results. There are very limited data on the correlation between degree of sarcopenia and muscle function, which has a nonlinear relationship in older non-cancer populations.

Conclusions: Loss of muscle mass is a significant contributor to morbidity in patients with lung cancer. Loss of muscle mass and function may predate clinically overt cachexia, underlining the importance of evaluating sarcopenia, rather than weight loss alone. Understanding this relationship and its associated factors will provide opportunities for focused intervention to improve clinical outcomes.

\section{INTRODUCTION}

Over the last decade, there has been increasing recognition of the clinical importance of

\section{Strengths and limitations of this study}

- Timely systematic review considering the increasingly recognised phenomenon of sarcopenia as it relates to cachexia.

- Evaluation of sarcopenia in lung cancer, as an example of a common cancer associated with poor outcomes and a significant prognostic impact.

- Limited to publications in English only.

sarcopenia as part of the cancer cachexia syndrome, and its impact has been evaluated in a wide range of malignancies including lung, breast, upper gastrointestinal, hepatocellular and colorectal cancers. ${ }^{1-5}$ The term sarcopenia is derived from the Greek meaning 'poverty of flesh' and is characterised by a triad of progressive loss of skeletal muscle mass, muscle strength and physical performance. ${ }^{6}$ It was originally described in the elderly non-cancer population, and is often now defined as a geriatric syndrome associated with functional impairment, increased risk of falls, fractures and reduced survival. $^{7-9}$ Sarcopenia becomes more prevalent with increase in age-of 1421 healthy adults aged 45 years or over, the overall prevalence was $15 \%$, rising to $64 \%$ over the age of $85 .{ }^{10}$ This loss of muscle mass is usually gradual and not necessarily associated with significant or sudden weight loss.

The detrimental effects of sarcopenia can also be seen in younger patients in association with muscle disuse, malnutrition or inflammatory conditions, particularly cancer. Interest in sarcopenia as a poor prognostic indicator in cancer is rising; in various populations with cancer sarcopenia is associated with poorer performance status (PS), ${ }^{1}$ reduced overall survival $^{11} 12$ and increased risk of chemotherapy toxicities. $^{2}{ }^{4}$ This interest is reflected in a recent international consensus on the definition of cancer cachexia, which established sarcopenia as a key diagnostic criterion. ${ }^{13}$ 
The hallmark of sarcopenia is low muscle mass, more specifically an appendicular skeletal muscle mass index of more than 2 SDs below the sex-specific mean of healthy adults (ie, $5.45 \mathrm{~kg} / \mathrm{m}^{2}$ for women and $7.26 \mathrm{~kg} / \mathrm{m}^{2}$ for men). ${ }^{8}$ However, central to the concept of sarcopenia is the recognition that overall neuromuscular function, rather than muscle mass alone, is essential for maintenance of independence. Therefore, when defining sarcopenia, it is vital to assess muscle strength, or physical performance, in addition to muscle mass, as the relationship between muscle mass and strength is non-linear. ${ }^{14} 15$

While many different techniques have been used to measure muscle mass and strength, few have been incorporated into routine assessment of the cancer population. The current gold standards are CT, MRI and dual-energy X-ray absorptiometry (DEXA) scans. CT and MRI allow precise differentiation between fat and other soft tissue including muscle and are therefore investigations of choice. CT focuses on a specific area of the body-for example, muscle cross-sectional area at the third lumbar vertebra-which can be related to whole body muscle mass. It is the current gold standard in body composition research and has the advantage that many patients will have CT scans as part of their diagnostic and treatment assessments. However, DEXA involves less radiation exposure compared with CT and accurately and precisely differentiates between lean and fat body compartments. ${ }^{16}$ More indirect techniques for measuring muscle mass include bioelectrical impedance analysis which is non-invasive but less accurate compared with DEXA. It includes a measure of organ mass other than skeletal muscle, but is easily performed in clinical settings. ${ }^{17}$ Measurements of mid-upper arm circumference and arm muscle area using skinfold thickness methods have also been used, ${ }^{18}$ although these assessments are less accurate and there exists considerable interobserver variability. Measurements of muscle strength in the literature have mainly centred around handgrip strength (HGS) and quadriceps strength, although in non-cancer elderly patients, functional assessments such as the Short Physical Performance Battery and sit-to-stand tests ${ }^{19}{ }^{20}$ have been shown to correlate with adverse outcomes.

From the literature it is clear that there is marked diversity in current clinical practice in assessing the degree of muscle loss in patients with cancer and in quantifying its functional implications. If the loss of muscle mass and strength have significant clinical implications for patients with cancer, then standardised, validated diagnostic thresholds are clearly needed. Furthermore, while the effects of sarcopenia have been extensively studied in the elderly patients, factors associated with loss of muscle mass and strength in cancer remain unclear. As the pathophysiological mechanisms responsible for loss of muscle mass in cancer differ, at least in part, from those in sarcopenia of ageing, it is necessary to evaluate cancer-specific causative factors and clinical implications.
We therefore undertook a systematic literature review to further understand the relationship between muscle function and muscle mass and its implications for research and clinical practice within the context of cancer. We limited the review to focus on lung cancer as an example of a common cancer, associated with poor outcomes, in which sarcopenia has been shown to have a significant prognostic impact. Lung cancer has a worldwide incidence rate of 1.61 million cases per year, ${ }^{21}$ and frequently presents in the advanced stages. Despite advances in anticancer therapies, survival benefits in patients with lung cancer over the past 30 years have been relatively small compared with those seen in breast, colorectal and prostate cancers. ${ }^{22}$ While reasons for this are complex, many patients with lung cancer are ineligible for radical treatment at presentation due to poor PS or comorbidity, while others fail to receive their intended treatment plan because of functional decline. ${ }^{23}{ }^{24}$ Non-small cell lung cancer (NSCLC) has a particularly strong association with loss of muscle massof 441 patients consecutively referred to a regional oncology service, $47 \%$ were found to be sarcopenic. ${ }^{25}$ This prevalence can be compared with $16 \%$ of a cohort of 471 survivors with breast cancer $^{26}$ and $39 \%$ in a cohort of 234 patients with preoperative colorectal cancer. ${ }^{5}$ We conducted this systematic review with this in mind.

\section{METHODS}

\section{Search strings and data sources}

We not only executed a broad literature search, including various terminologies used to describe loss of muscle mass, but also specifically used 'sarcopenia' as a multipurpose field search term. Recognising that changes in muscle mass may impact on muscle strength and physical function, we included these terms in our search. We united two search strings: loss of muscle mass (and its implications) and lung cancer (see table 1). The search was limited to English language and humans, with a publication date from 1946 to October 2013. We used the same search strings to develop strategies in the following five databases in order to ensure maximal coverage: MEDLINE, MEDLINE In-Process, EMBASE, AMED and the Cochrane library.

\section{Article retrieval}

Citations were independently screened by two researchers (JCo and SN) and included for initial analysis if they described muscle mass measurements or body composition in patients with lung cancer. Both prospective and retrospective original articles were included, but conference abstracts, citations without abstracts, case reports, review articles and opinion pieces were excluded. All studies that had a cohort of patients with lung cancer were included, even if there were patient groups with other cancer types analysed. Retrieved articles were searched for additional relevant references. 


\begin{tabular}{ll} 
Table 1 Search strings and terms \\
\hline Search strings & Search terms \\
\hline Loss of muscle & Sarcopenia OR \\
mass & Muscle atrophy OR \\
& Muscle weakness OR \\
& Muscle mass OR \\
& Muscle wasting OR \\
& Muscle loss OR \\
& Weight loss OR \\
& Muscle strength OR \\
& Physical fitness OR \\
& Physical exertion OR \\
& Activities of daily living OR \\
& Cachexia \\
& AND \\
& Lung (neoplasm OR malignancy OR \\
& tumour) \\
& Pleural (neoplasm OR malignancy OR \\
& tumour) \\
\hline
\end{tabular}

\section{Inclusion criteria and data extraction}

The selected articles were reviewed in full by two independent researchers (JCo and $\mathrm{SN}$ ) for consideration of inclusion in the review. Where discordance in selection was observed, the article in question was discussed until consensus was reached. As there were many articles describing muscle mass as part of routine anthropometry or body composition assessments, without directly exploring it, we only included articles in which muscle mass was explored as an outcome measure. Data including patient characteristics and numbers, histological subtype and TNM (tumour, node, metastasis) stage were collated and tabulated (tables 2 and 3). We also noted units of muscle mass measurements, and techniques used to measure these.

\section{RESULTS}

Using our broad search terms in five databases, we found an initial 5726 citations, from which we identified 64 potentially relevant articles. Three further potential articles $^{27-29}$ were identified from the references of these articles. From these, we excluded 11 abstracts with no published articles, 2 further abstracts that did not mention muscle mass, ${ }^{30}{ }^{31}$ and a systematic review of cancer cachexia. ${ }^{32}$ Of the 53 final articles, we excluded a further 13 articles which mentioned muscle mass in baseline anthropometry details but did not use it as an outcome measure, ${ }^{33-45} 4$ articles which described weight loss rather than loss of muscle mass, ${ }^{46-49}$ and 1 article describing the same results obtained from the same patient population as another article ${ }^{50}$ with slightly different secondary endpoints. ${ }^{51}$ During the process of data extraction, a number of recurring themes became apparent. We have presented these under two main headings -factors associated with loss of muscle mass, and degree of loss of muscle mass and physical functioning.
A number of studies explored multiple variables associated with loss of muscle mass, and are therefore mentioned under more than one heading.

For the final analysis, 4 randomised controlled studies, 17 cross-sectional studies and 14 longitudinal studies met the established criteria: 35 articles in total. Muscle mass data were reported variously as fat-free mass (FFM), body cell mass, lean body mass, appendicular skeletal muscle mass, smooth muscle area at the levels of lumbar vertebra L3 and thoracic vertebra T4, mid-upper arm circumference and arm muscle area. Notably, most studies described muscle mass in more than one way. Muscle function was described as HGS and/or quadriceps strength, ${ }^{17}{ }^{27}{ }^{52-54}$ intensity of physical activity, ${ }^{55}$ patient-reported physical functioning ${ }^{28}$ and muscle strength and physical performance. ${ }^{56}$

As the studies in our review expressed muscle mass in different ways, we have used the term FFM or loss of muscle mass in the body of our article in order to allow direct comparison. In addition, where the studies in this review defined a patient group as having sarcopenia, they did so based on loss of muscle mass alone, without evaluation of muscle strength or performance. This needs to be borne in mind wherever the term sarcopenia is used throughout this review.

\section{Factors associated with loss of muscle mass}

In this review, many studies found that those with cachexia and/or weight loss also had coexistent loss of muscle mass. ${ }^{25} 285867687174$ Despite this, none of the studies prospectively evaluated the impact of loss of muscle mass on cancer outcomes, specifically relating to treatment. Three studies explored the loss of muscle mass comparing men and women, finding that a significantly greater percentage of men were affected, ${ }^{25} 64$ and that they exhibited a decreasing trend in FFM after chemotherapy compared with baseline, whereas women did not. ${ }^{29}$ Reflecting the process of loss of muscle mass in the different stages of cancer, a study of 60 patients with preoperative NSCLC with stage I and II disease showed no difference in FFM compared with controls, ${ }^{27}$ whereas in a cohort of 352 patients with advanced cancer, $84 \%$ of those with cachexia had a reduced FFM. ${ }^{74}$

The pathophysiology of loss of muscle mass in patients with lung cancer is complex, as illustrated in the diversity of articles exploring this. Low FFM has been associated with low albumin and high acute phase protein concentrations, ${ }^{57} 5871$ reflecting the inflammatory pathways involved. Abnormal protein metabolism is implicated in the development of sarcopenia; however, in this review, neither anabolic ${ }^{58}$ nor proteolytic pathways ${ }^{59} 55$ had any consistent effect on loss of muscle mass. The exception to this was the lysosomal protease cathepsin-B, which was significantly increased in depleted FFM patients. ${ }^{59}$ The pathophysiology may also differ depending on disease stage and cachexia phase. There is some evidence, for example, that in patients with precachectic NSCLC, despite weight loss, the ubiquitin-proteasome 
Table 2 Loss of muscle mass as outcome measures and factors associated with it

\begin{tabular}{|c|c|c|c|c|c|c|c|c|}
\hline \multirow[b]{2}{*}{ First author (year) } & \multicolumn{4}{|l|}{ Patients } & \multicolumn{2}{|l|}{ Study } & \multirow[b]{2}{*}{ Comparison } & \multirow[b]{2}{*}{ Result } \\
\hline & $\mathrm{n}(\mathrm{M} / \mathrm{F})$ & Tumour, stage & $\begin{array}{l}\text { Muscle mass } \\
\text { measurement(s) }\end{array}$ & $\begin{array}{l}\text { Method of } \\
\text { measurement }\end{array}$ & Design & Controls & & \\
\hline McMillan $(2001)^{57}$ & $40(40 / 0)$ & $\begin{array}{l}\text { NSCLC } n=11 \text {, } \\
\text { upper } \mathrm{Gl} n=22 \text {, } \\
\text { colon } n=7 \\
\text { All locally } \\
\text { advanced or } \\
\text { metastatic }\end{array}$ & $\mathrm{BCM}$ & $\begin{array}{l}\text { Total body } \\
\text { potassium }\end{array}$ & $\begin{array}{l}\text { Cross } \\
\text { sectional }\end{array}$ & Nil & $\begin{array}{l}\text { The inter-relationship } \\
\text { between albumin, body } \\
\text { cell mass and the } \\
\text { systemic inflammatory } \\
\text { response }\end{array}$ & $\begin{array}{l}\text { Albumin concentrations } \\
\text { correlated with } B C M \\
(r=0.686, p<0.001) \text { and } \\
\text { negatively correlated } \\
\text { with CRP }(r=-0.545, \\
p<0.001)\end{array}$ \\
\hline Crown $(2002)^{58}$ & $\begin{array}{l}30(\mathrm{NR} / \\
\mathrm{NR})\end{array}$ & $\begin{array}{l}\text { NSCLC in all } \\
\text { Inoperable, stage } \\
\text { NR }\end{array}$ & FFM, MUAC & $\begin{array}{l}\text { BIA, upper } \\
\text { arm } \\
\text { measurements }\end{array}$ & $\begin{array}{l}\text { Case-control, } \\
\text { longitudinal } \\
\text { over } 2 \text { years }\end{array}$ & $\mathrm{n}=30 \mathrm{HV}$ & $\begin{array}{l}\text { ILGF system and } \\
\text { cancer cachexia }\end{array}$ & $\begin{array}{l}\text { More LC than HV had } \\
\text { MAMC in the lowest } \\
\text { quartile }(p<0.05) \text { at } \\
\text { baseline, } \\
\text { Male LC patients had } \\
\text { lower FFM than male } \\
\text { HV }(p<0.05) \text { at } \\
\text { baseline, } \\
\text { No significant } \\
\text { longitudinal trend } \\
\text { observed in IGFBP-3 } \\
\text { and IL- } 6 \text { and nutritional } \\
\text { status, } p=N S\end{array}$ \\
\hline Jagoe $(2002)^{59}$ & $36(27 / 9)$ & $\begin{array}{l}\text { Mix of NSCLC } \\
\text { and SCLC } \\
\text { Stage } 1-21 \\
\text { Stage } 2-6 \\
\text { Stage 3-6 } \\
\text { Stage } 4-2\end{array}$ & FFMi & $\begin{array}{l}\text { BIA, Four } \\
\text { skinfold } \\
\text { method, \% } \\
\text { BFMAMA }\end{array}$ & $\begin{array}{l}\text { Cross } \\
\text { sectional }\end{array}$ & $\begin{array}{l}\mathrm{n}=10 \\
\text { patients } \\
\text { referred for } \\
\text { thoracotomy } \\
\text { for } \\
\text { non-malignant } \\
\text { conditions }\end{array}$ & $\begin{array}{l}\text { Ubiquitin-proteasome } \\
\text { and lysosomal } \\
\text { proteolytic pathway } \\
\text { gene expression in LC } \\
\text { and association with } \\
\text { LMM }\end{array}$ & $\begin{array}{l}\text { Cathepsin B } \\
\text { expression in LC } \\
\text { inversely related to } \\
\text { FFMi, } p=0.003 \text {; } \\
\text { Cathepsin-B } \\
\text { expression increased } \\
\text { in 'depleted FFMi } \\
\text { cancer patients' vs } \\
\text { controls } p=0.003 \text {; } \\
\text { No relationship } \\
\text { between cathepsin B } \\
\text { expression and } \\
\text { \%BFMAMA, } p=N S\end{array}$ \\
\hline Wieland (2007) & $\begin{array}{l}286(\mathrm{NR} / \\
\mathrm{NR})\end{array}$ & $\begin{array}{l}\text { NSCLC } n=181 \text {, } \\
\text { stage IIIB or IV }\end{array}$ & SMA at T4 & CT at $\mathrm{T} 4$ & Longitudinal & $\mathrm{n}=7 \mathrm{HV}$ & $\begin{array}{l}\text { Establish prevalence of } \\
\text { PIF in patients with } \\
\text { cancer, and its } \\
\text { association with } \\
\text { muscle loss }\end{array}$ & $\begin{array}{l}\text { In patients with } \\
\text { NSCLC: } \\
\text { PIF unrelated to } \\
\text { survival and muscle } \\
\text { loss, p=NS; } \\
\text { PIF positive patients } \\
\text { rate of loss of muscle } \\
\text { mass per } 100 \text { days }\end{array}$ \\
\hline
\end{tabular}




\begin{tabular}{|c|c|c|c|c|c|c|c|c|}
\hline \multirow[b]{2}{*}{ First author (year) } & \multicolumn{4}{|l|}{ Patients } & \multicolumn{2}{|l|}{ Study } & \multirow[b]{2}{*}{ Comparison } & \multirow[b]{2}{*}{ Result } \\
\hline & $n(M / F)$ & Tumour, stage & $\begin{array}{l}\text { Muscle mass } \\
\text { measurement(s) }\end{array}$ & $\begin{array}{l}\text { Method of } \\
\text { measurement }\end{array}$ & Design & Controls & & \\
\hline & & & & & & & & $\begin{array}{l}-3.4 \pm 2.1 \% \text { vs PIF } \\
\text { negative patients } \\
-2.4 \pm 1.7 \%, p=N S\end{array}$ \\
\hline $\begin{array}{l}\text { Martinez-Hernandez } \\
(2012)^{61}\end{array}$ & $21(19 / 2)$ & $\begin{array}{l}\mathrm{LC} n=13, \mathrm{GI} \\
\text { cancer } n=6 \text {, } \\
\text { Other cancer } n=2 \\
\text { Stage according } \\
\text { to tumour group } \\
\text { NR }\end{array}$ & FFM & $\mathrm{BIA}$ & Longitudinal & $\mathrm{n}=8 \mathrm{HV}$ & $\begin{array}{l}\text { The role of IL-15) in } \\
\text { patients with cachectic } \\
\text { cancer }\end{array}$ & $\begin{array}{l}\text { At weeks } 4 \text { and } 8 \text {, } \\
\text { patients with cancer } \\
\text { lost FFM in tandem } \\
\text { with decreasing IL-15 } \\
\text { levels, } r=0.514 \text { and } \\
r=0.535 \text {, both } p<0.05\end{array}$ \\
\hline $\begin{array}{l}\text { Op den Kamp } \\
(2012)^{55}\end{array}$ & $16(15 / 1)$ & $\begin{array}{l}\text { NSCLC in all } \\
\text { Stage I-II-11 } \\
\text { Stage IIIA-2 } \\
\text { Stage IIIB-3 }\end{array}$ & FFMi & DEXA & $\begin{array}{l}\text { Cross } \\
\text { sectional }\end{array}$ & $\mathrm{n}=10 \mathrm{HV}$ & $\begin{array}{l}\text { Skeletal muscle NF-кB } \\
\text { and ubiquitin } \\
\text { proteasome system } \\
\text { activity in precachexia }\end{array}$ & $\begin{array}{l}\text { FFMi no significant } \\
\text { difference in } \\
\text { precachectic cancer vs } \\
\text { controls, } p=N S \text {; } \\
\text { NF- } \mathrm{B} \text {, UPS E3-ligase } \\
\text { and } 26 S \text { proteasome } \\
\text { activity not raised in } \\
\text { patients with } \\
\text { precachectic cancer, } \\
\text { all } p=N S\end{array}$ \\
\hline Vigano $(2009)^{52}$ & $\begin{array}{l}N=172 \\
(101 / 71)\end{array}$ & $\begin{array}{l}\text { NSCLC } n=64 \text {, All } \\
\text { stage III and IV. } \\
\text { Metastatic GI } \\
\text { cancer } n=108\end{array}$ & LBM, ALM & DEXA $(n=64)$ & $\begin{array}{l}\text { Cross } \\
\text { sectional }\end{array}$ & Nil & $\begin{array}{l}\text { ACE gene } \\
\text { polymorphism } \\
\text { (insertion }^{2}-\text { II, insertion/ } \\
\text { deletion-ID, } \\
\text { deletion }^{2} \text {-DD) on } \\
\text { nutritional status }\end{array}$ & $\begin{array}{l}\text { Trend }(p=0.07) \\
\text { towards lower LBM in } \\
\text { ID compared to } \\
\text { II groups }\end{array}$ \\
\hline $\begin{array}{l}\text { Op den Kamp } \\
(2013)^{62}\end{array}$ & $26(17 / 9)$ & $\begin{array}{l}\text { NSCLC } \\
\text { Stage IIIB-10 } \\
\text { Stage IV-16 }\end{array}$ & FFMi, AMMi & DEXA & $\begin{array}{l}\text { Cross } \\
\text { sectional }\end{array}$ & $\mathrm{n}=10 \mathrm{HV}$ & $\begin{array}{l}\text { Expression of } \\
\text { signalling molecules in } \\
\text { protein metabolism in } \\
\text { LC cachexia }\end{array}$ & $\begin{array}{l}\text { AMMi } 20 \% \text { lower in } \\
\text { cachectic group } \\
\text { compared with } \\
\text { controls, } p<0.05 \text {; } \\
\text { Akt concentration } \\
\text { increased in cachectic } \\
\text { group }(p<0.05) \text {, but no } \\
\text { downstream signal } \\
\text { phosphorylation, that } \\
\text { is, impaired anabolic } \\
\text { activity }\end{array}$ \\
\hline Harvie $(2003)^{29}$ & $50(32 / 18)$ & $\begin{array}{l}\text { NSCLC in all, } \\
\text { stage III and IV }\end{array}$ & FFM & $\begin{array}{l}\text { Four skinfold } \\
\text { method }\end{array}$ & Longitudinal & Nil & $\begin{array}{l}\text { Exploration of } \\
\text { gender-specific } \\
\text { differences in body } \\
\text { composition and REE }\end{array}$ & $\begin{array}{l}\text { Trend for FFM to } \\
\text { decrease }(p=0.063) \\
\text { and FFM decreased } \\
(p<0.05) \text { in men after }\end{array}$ \\
\hline
\end{tabular}




\begin{tabular}{|c|c|c|c|c|c|c|c|c|}
\hline \multirow[b]{2}{*}{ First author (year) } & \multicolumn{4}{|l|}{ Patients } & \multicolumn{2}{|l|}{ Study } & \multirow[b]{2}{*}{ Comparison } & \multirow[b]{2}{*}{ Result } \\
\hline & n (M/F) & Tumour, stage & $\begin{array}{l}\text { Muscle mass } \\
\text { measurement(s) }\end{array}$ & $\begin{array}{l}\text { Method of } \\
\text { measurement }\end{array}$ & Design & Controls & & \\
\hline & & & & & & & $\begin{array}{l}\text { prechemotherapy and } \\
\text { postchemotherapy }\end{array}$ & $\begin{array}{l}\text { chemotherapy. No } \\
\text { significant difference in } \\
\text { FFM or REE in women }\end{array}$ \\
\hline Harvie $(2005)^{63}$ & $43(28 / 15)$ & $\begin{array}{l}\text { NSCLC in all, } \\
\text { stage III and IV. } \\
\text { Alongside this } \\
\text { metastatic breast } \\
\text { and melanoma } \\
\text { patients } \\
\text { evaluated } \\
\text { separately }\end{array}$ & FFM & $\begin{array}{l}\text { Four skinfold } \\
\text { method }\end{array}$ & Longitudinal & Nil & $\begin{array}{l}\text { Relationship between } \\
\text { energy intake, REE } \\
\text { and acute phase } \\
\text { response vs changes } \\
\text { in body composition } \\
\text { over course of } \\
\text { chemotherapy }\end{array}$ & $\begin{array}{l}\text { No significant change } \\
\text { in FFM over the course } \\
\text { of chemotherapy, and } \\
\text { no significant } \\
\text { relationship with } \\
\text { energy intake, REE or } \\
\text { CRP (all } p=N S \text { ) }\end{array}$ \\
\hline Bovio $(2008)^{64}$ & $\begin{array}{l}144(92 / \\
52)\end{array}$ & $\begin{array}{l}\mathrm{LC} n=46, \text { colon } \\
n=22, \text { HCC } n=11 \text {, } \\
\text { other } n=65 \\
\text { Stage NR }\end{array}$ & AMA & $\begin{array}{l}\text { Upper arm } \\
\text { measurements }\end{array}$ & $\begin{array}{l}\text { Cross } \\
\text { sectional }\end{array}$ & Nil & $\begin{array}{l}\text { Evaluation of nutritional } \\
\text { status in patients with } \\
\text { advanced cancer }\end{array}$ & $\begin{array}{l}63 \% \text { men vs } 19 \% \\
\text { women had } A M A<5 \text { th } \\
\text { centile }(p<0.01)\end{array}$ \\
\hline Baracos $(2010)^{25}$ & $\begin{array}{l}441(229 / \\
212)\end{array}$ & $\begin{array}{l}\text { NSCLC in all } \\
\text { Stage III-206 } \\
\text { Stage IV-235 }\end{array}$ & SMA at L3 & CT of L3 & $\begin{array}{l}\text { Cross } \\
\text { sectional }\end{array}$ & Nil & $\begin{array}{l}\text { The use of CT images } \\
\text { in evaluating body } \\
\text { composition in NSCLC }\end{array}$ & $\begin{array}{l}61.1 \% \text { men in cohort } \\
\text { were sarcopenic, } \\
31.3 \% \text { of women } \\
\text { sarcopenic, } p<0.001\end{array}$ \\
\hline Martin (2013) ${ }^{65}$ & $\begin{array}{l}1473(828 / \\
645)\end{array}$ & $\begin{array}{l}\text { Colorectal cancer } \\
n=773, L C \\
n=440, \text { other } G I \\
\text { cancer } n=260 \\
\text { Stage according } \\
\text { to cancer NR }\end{array}$ & SMA at L3, SMAi & CT of L3 & Longitudinal & Nil & $\begin{array}{l}\text { Prognostic significance } \\
\text { of weight loss, muscle } \\
\text { mass index and } \\
\text { muscle attenuation }\end{array}$ & $\begin{array}{l}\text { Concordance model } \\
\text { using variables of BMI, } \\
\text { weight loss, MI and } \\
\text { MA found a } \\
\text { concordance statistic } \\
\text { (predictive accuracy of } \\
\text { survival) of } 0.92 \\
\text { Regardless of BMI, pts } \\
\text { with weight loss, low } \\
\text { MI and MA reduced } \\
\text { survival ( } 8.4 \text { months), } \\
\text { compared to those with } \\
\text { none of these features } \\
\text { ( } 28.4 \text { months), p<0.001 }\end{array}$ \\
\hline Prado $(2013)^{66}$ & $\begin{array}{l}368(216 / \\
152)\end{array}$ & $\begin{array}{l}\text { NSCLC } n=242 \\
\text { Gl tract cancer } \\
n=126\end{array}$ & SMA at L3 & CT of L3 & Longitudinal & Nil & $\begin{array}{l}\text { Clinical course of } \\
\text { skeletal muscle } \\
\text { wasting in advanced } \\
\text { cancer }\end{array}$ & $\begin{array}{l}\text { Being }<90 \text { days from } \\
\text { death increases risk of } \\
\text { muscle loss, OR } 2.67 \text {, } \\
p=0.002 ; \text { and } \\
\text { decreases chance of }\end{array}$ \\
\hline
\end{tabular}




\begin{tabular}{|c|c|c|c|c|c|c|c|c|}
\hline \multirow[b]{2}{*}{ First author (year) } & \multicolumn{4}{|l|}{ Patients } & \multicolumn{2}{|l|}{ Study } & \multirow[b]{2}{*}{ Comparison } & \multirow[b]{2}{*}{ Result } \\
\hline & $\mathrm{n}(\mathrm{M} / \mathrm{F})$ & Tumour, stage & $\begin{array}{l}\text { Muscle mass } \\
\text { measurement(s) }\end{array}$ & $\begin{array}{l}\text { Method of } \\
\text { measurement }\end{array}$ & Design & Controls & & \\
\hline & & & & & & & & $\begin{array}{l}\text { muscle gain, } O R=0.37, \\
p=0.002\end{array}$ \\
\hline Hansell $(1986)^{67}$ & $98(63 / 35)$ & $\begin{array}{l}\text { Colorectal cancer } \\
n=55 \text {, gastric } \\
\text { cancer } n=24, L C \\
n=12 \text {, other } \\
\text { cancer } n=7 \\
\text { Stage NR }\end{array}$ & LBM, MUAC & $\begin{array}{l}\text { Tritiated } \\
\text { saline, upper } \\
\text { arm } \\
\text { measurements }\end{array}$ & $\begin{array}{l}\text { Cross } \\
\text { sectional }\end{array}$ & $\begin{array}{l}\mathrm{n}=38 \\
\text { non-malignant } \\
\text { illnesses }\end{array}$ & $\begin{array}{l}\text { REE in weight-losing } \\
\text { patients with cancer } \\
\text { WLC=weight-losing } \\
\text { patients with cancer, } \\
\text { WSC=weight-stable } \\
\text { patients with cancer, } \\
\text { WSC on=weight-stable } \\
\text { controls }\end{array}$ & $\begin{array}{l}\text { WLC compared to } \\
\text { WSC had lower LBM } \\
\text { ( } p<0.005) \text {; } \\
\text { WLC compared to } \\
\text { WSC and WSC on } \\
\text { lower MAMC } \\
\text { ( } p<0.0005) \text {; } \\
\text { WLC had increased } \\
\text { REE/kg bodyweight } \\
\text { compared with both } \\
\text { WS groups ( } p<0.005) \text {; } \\
\text { No significant } \\
\text { difference when REE } \\
\text { is expressed in terms } \\
\text { of kg LBM; } \\
\text { WLC had positive } \\
\text { relationship with REE, } \\
r=0.83, p<0.001\end{array}$ \\
\hline Fredrix $(1990)^{68}$ & $\begin{array}{l}39 \text { (GCR } \\
13 / 9, \text { LC } \\
16 / 1)\end{array}$ & $\begin{array}{l}\mathrm{LC} n=17 \\
\text { GCR-n=22 } \\
\text { Stage NR }\end{array}$ & FFM & $\mathrm{BIA}$ & $\begin{array}{l}\text { Cross } \\
\text { sectional }\end{array}$ & $n=40$ healthy & REE and weight loss & $\begin{array}{l}\text { FFM: LC 50.4 } 58.9 \text {, } \\
\text { Controls } 51.1 \pm 9.6 \text {, } \\
p=N S \text {; } \\
\text { REE/FFM: LC } \\
33.5 \pm 5.4 \text {, Controls } \\
29.6 \pm 2.9, p<0.01\end{array}$ \\
\hline $\begin{array}{l}\text { Staal-van den Brekel } \\
(1997)^{69}\end{array}$ & $12(10 / 2)$ & All SCLC & FFM & $\mathrm{BIA}$ & Longitudinal & Nil & $\begin{array}{l}\text { Assess REE and } \\
\text { systemic inflammation } \\
\text { prechemotherapy and } \\
\text { postchemotherapy }\end{array}$ & $\begin{array}{l}\text { No change in FFM } \\
\text { postchemotherapy } \\
\text { ( } p=N S) \text {. Absolute REE } \\
\text { and REE adjusted for } \\
\text { FFM decreased } \\
\text { postchemotherapy } \\
(p<0.005)\end{array}$ \\
\hline Simons $(1997)^{70}$ & $21(21 / 0)$ & $\begin{array}{l}\text { NSCLC } n=19 \\
\text { Stage I-3 } \\
\text { Stage III-5 } \\
\text { Stage IV-11 }\end{array}$ & FFM, FFMi & DEXA & $\begin{array}{l}\text { Cross } \\
\text { sectional }\end{array}$ & Nil & $\begin{array}{l}\text { Relationship between } \\
\text { DL expression, body } \\
\text { composition and REE }\end{array}$ & $\begin{array}{l}\text { DL vs non-DL no } \\
\text { significant difference } \\
\text { between groups with } \\
\text { regards FFM, FFMi, }\end{array}$ \\
\hline
\end{tabular}




\begin{tabular}{|c|c|c|c|c|c|c|c|c|}
\hline Scott $(2001)^{72}$ & $12(12 / 0)$ & $\begin{array}{l}\text { NSCLC in all, } \\
\text { locally advanced }\end{array}$ & FFM BCM I BM & $\begin{array}{l}\text { Total body } \\
\text { potassium }\end{array}$ & Longitudinal & $\begin{array}{l}\mathrm{n}=7 \text {, healthy } \\
\text { participants }\end{array}$ & $\begin{array}{l}\text { Inter-relationship } \\
\text { between systemic } \\
\text { inflammation and REE } \\
\text { preonset and postonset } \\
\text { of weight loss }\end{array}$ & $\begin{array}{l}\text { Karnofsky PS, } p=0.02 \\
\text { Cancer group had } \\
\text { lower REE }(p<0.05) \\
\text { and BCM }(p<0.001) \text {. } \\
\text { Cancer group REE } \\
\text { adjusted for BCM } \\
\text { correlated with CRP } \\
\text { concentrations } \\
\text { (r=0.753, } p<0.01)\end{array}$ \\
\hline Jatoi $(2001)^{73}$ & $18(10 / 8)$ & $\begin{array}{l}\text { NSCLC in all } \\
\text { Stage IA-6 } \\
\text { Stage IB-3 } \\
\text { Stage IIB-3 } \\
\text { Stage IIIA-4 } \\
\text { Stage IIIB-2 }\end{array}$ & FFM, BCM, LBM & $\begin{array}{l}\text { DEXA, } \\
\text { Potassium-40, } \\
\text { tritium dilution }\end{array}$ & $\begin{array}{l}\text { Cross } \\
\text { sectional }\end{array}$ & $\mathrm{n}=18, \mathrm{HV}$ & $\begin{array}{l}\text { REE in non-metastatic } \\
\text { NSCLC }\end{array}$ & $\begin{array}{l}\text { REE in cancer vs } \\
\text { controls significantly } \\
\text { raised when adjusted } \\
\text { for } L B M, p=0.001 ; \\
\text { and also when } \\
\text { adjusted for } B C M \text {, } \\
p=0.032\end{array}$ \\
\hline Jagoe $(2001)^{27}$ & $60(43 / 17)$ & LC in all & $\begin{array}{l}\text { FFM, MAMC, } \\
\text { BFMAMA }\end{array}$ & $\begin{array}{l}\text { BIA, four } \\
\text { skinfold } \\
\text { thickness, } \\
\text { upper arm } \\
\text { measurements }\end{array}$ & $\begin{array}{l}\text { Cross } \\
\text { sectional }\end{array}$ & $\begin{array}{l}\mathrm{n}=22, \text { mild } \\
\text { COPD }\end{array}$ & $\begin{array}{l}\text { Nutritional status of } \\
\text { patients undergoing LC } \\
\text { operations }\end{array}$ & $\begin{array}{l}\text { No difference in FFMi } \\
\text { and BFMAMA } \\
\text { comparing LC and } \\
\text { controls, all } p=N S\end{array}$ \\
\hline Sarhill $(2003)^{74}$ & $\begin{array}{l}\mathrm{n}=352 \text { but } \\
\mathrm{LC} \text { only } \\
18 \% \text { of } \\
\text { cohort }()\end{array}$ & NR & MUAC, AMA & $\mathrm{BIA}(\mathrm{n}=329)$ & $\begin{array}{l}\text { Cross } \\
\text { sectional }\end{array}$ & Nil & $\begin{array}{l}\text { Prospective evaluation } \\
\text { of nutritional status in } \\
\text { advanced cancer }\end{array}$ & $\begin{array}{l}\text { Cachexia group vs } \\
\text { non-cachexia group, } \\
\text { reduced AMA in } 84 \% \\
\text { vs } 69 \%, p=0.037\end{array}$ \\
\hline Prado $(2008)^{1}$ & $\begin{array}{l}n=250 \text {, } \\
\text { with LC } 60 \\
(24 \%) \text { of }\end{array}$ & $\begin{array}{l}\text { TNM for cohort } \\
\text { Stage I-24 } \\
\text { Stage II-56 }\end{array}$ & $\begin{array}{l}\text { SMA and SMAi } \\
\text { at L3 }\end{array}$ & CT of L3 & $\begin{array}{l}\text { Cross } \\
\text { sectional }\end{array}$ & Nil & $\begin{array}{l}\text { Prevalence of } \\
\text { sarcopaenic obesity } \\
\text { and chemotherapy }\end{array}$ & $\begin{array}{l}\text { SMA in OS } \\
128.1 \pm 29.1, \text { ONons } \\
160 \pm 38.1, p<0.0001\end{array}$ \\
\hline
\end{tabular}




\begin{tabular}{|c|c|c|c|c|c|c|c|c|}
\hline \multirow[b]{2}{*}{ First author (year) } & \multicolumn{4}{|l|}{ Patients } & \multicolumn{2}{|l|}{ Study } & \multirow[b]{2}{*}{ Comparison } & \multirow[b]{2}{*}{ Result } \\
\hline & n (M/F) & Tumour, stage & $\begin{array}{l}\text { Muscle mass } \\
\text { measurement(s) }\end{array}$ & $\begin{array}{l}\text { Method of } \\
\text { measurement }\end{array}$ & Design & Controls & & \\
\hline & $\begin{array}{l}\text { cohort } \\
(136 / 114)\end{array}$ & $\begin{array}{l}\text { Stage III-74 } \\
\text { Stage IV-96 }\end{array}$ & & & & & $\begin{array}{l}\text { toxicity in this cohort } \\
\text { OS=obese sarcopaenic } \\
\text { ONonS=obese } \\
\text { non-sarcopaenic }\end{array}$ & $\begin{array}{l}\text { SMAi in OS } 43.3 \pm 6.3 \text {, } \\
\text { ONonS } 56.4 \pm 9.9 \text {; } \\
\text { Median survival assoc } \\
\text { with sarcopenia log } \\
\text { rank, } p<0.0001, \text { OS } \\
11.3 \text { months and } \\
\text { ONonS } 21.6 \text { months, } \\
p<0.0001\end{array}$ \\
\hline Kilgour $(2010)^{53}$ & $\begin{array}{l}n=84, \text { with } \\
\text { LC } 16 \\
(19 \%) \text { of } \\
\text { cohort }(48 / \\
36)\end{array}$ & $\begin{array}{l}\text { Metastatic } 57 \% \text {, } \\
\text { locally advanced } \\
43 \%, \text { stage NR }\end{array}$ & SMMI, ALM & DEXA & $\begin{array}{l}\text { Cross } \\
\text { sectional }\end{array}$ & Nil & $\begin{array}{l}\text { Relationship of fatigue } \\
\text { to muscle mass and } \\
\text { strength }\end{array}$ & $\begin{array}{l}\text { Brief fatigue index } \\
\text { associated with SMMI } \\
(95 \% \mathrm{Cl}-8.4 \text { to }-1.3) \\
p<0.01 \text {, and } \\
\text { sarcopenia, } p<0.01\end{array}$ \\
\hline $\begin{array}{l}\text { Peddle-Mclntyre } \\
(2012)^{56}\end{array}$ & $17(7,10)$ & $\begin{array}{l}\text { NSCLC } n=16 \\
\text { Stage I-II-11 } \\
\text { Stage III-5 } \\
\text { Limited stage } \\
\text { SCLC } n=1\end{array}$ & LBM, ALM & DEXA & $\begin{array}{l}\text { Longitudinal, } \\
\text { duration } \\
10 \text { weeks }\end{array}$ & Nil & $\begin{array}{l}\text { Resistance exercise } \\
\text { training efficacy and } \\
\text { feasibility in LC } \\
\text { survivors }\end{array}$ & $\begin{array}{l}\text { LBM and ALM no } \\
\text { change from baseline } \\
\text { to post training, all } \\
p=N S\end{array}$ \\
\hline Bauer $(2005)^{75}$ & $\begin{array}{l}n=7, \text { with } \\
\text { NSCLC } 2 \\
(28.6 \%) \text { of } \\
\text { cohort }\end{array}$ & $\begin{array}{l}\text { Adenocarcinoma } \\
\text { pancreas } n=5 \text {, } \\
\text { NSCLC } n=2 \\
\text { Stage NR }\end{array}$ & LBM & $\begin{array}{l}\text { Deuterium } \\
\text { dilution }\end{array}$ & $\begin{array}{l}\text { Longitudinal, } \\
\text { duration } \\
10 \text { weeks }\end{array}$ & Nil & $\begin{array}{l}\text { Effect of nutrition } \\
\text { counselling and EPA } \\
\text { supplements on body } \\
\text { composition }\end{array}$ & $\begin{array}{l}\text { Change in LBM post } \\
\text { intervention, } p=N S\end{array}$ \\
\hline Fearon $(2006)^{28}$ & $\begin{array}{l}518(355 / \\
163)\end{array}$ & $\begin{array}{l}\text { LC } n=231 \\
\text { Upper GI cancer } \\
n=198 \\
\text { Other GI cancer } \\
n=89 \\
\text { Stage NR }\end{array}$ & LBM & $\mathrm{BIA}$ & $\begin{array}{l}\mathrm{RCT} \text { (double } \\
\text { blind, placebo } \\
\text { controlled, } \\
\text { randomised) }\end{array}$ & Nil & $\begin{array}{l}\text { Effect of } 2 \mathrm{~g} \text { and } 4 \mathrm{~g} \\
\text { doses of EPA diester } \\
\text { vs placebo in the } \\
\text { process of cachexia }\end{array}$ & $\begin{array}{l}\text { Group given } 2 \mathrm{~g} \text { EPA } \\
\text { gained mean } 0.9 \mathrm{~kg} \\
\text { LBM and group given } \\
4 \mathrm{~g} \text { EPA lost mean } \\
0.1 \mathrm{~kg} \text { LBM compared } \\
\text { to placebo ( } \mathrm{p}=\mathrm{NS} \text { ) }\end{array}$ \\
\hline Tozer $(2008)^{54}$ & $\begin{array}{l}66(49 / 17) \\
\text { only } 35 \\
\text { completed } \\
\text { study }\end{array}$ & $\begin{array}{l}\text { All LC } \\
\text { Stage NR }\end{array}$ & $\mathrm{BCM}$ & NR & $\begin{array}{l}\text { RCT (double } \\
\text { blind, placebo } \\
\text { controlled, } \\
\text { randomised) }\end{array}$ & Nil & $\begin{array}{l}\text { Effect of cysteine-rich } \\
\text { protein supplement on } \\
\text { body weight and body } \\
\text { cell mass }\end{array}$ & $\begin{array}{l}\text { Cysteine group }+11.55 \\
\pm 18.05 \% \text { vs control } \\
\text { group } \\
-5.47 \pm 34.63 \% \text { after } \\
\text { treatment }(p=0.01) \text {, } \\
\text { and compared to } \\
\text { baseline }(p=0.02)\end{array}$ \\
\hline Murphy $(2010)^{76}$ & $41(19 / 22)$ & $\begin{array}{l}\text { NSCLC in all } \\
\text { Stage I-2 } \\
\text { Stage II-2 }\end{array}$ & SMA at L3 & CT of L3 & $\begin{array}{l}\text { Longitudinal, } \\
\text { cohort study }\end{array}$ & Nil & $\begin{array}{l}\text { Relationship between } \\
\text { muscle mass, rate of }\end{array}$ & $\begin{array}{l}\text { Sarcopenia at baseline } \\
\text { in } 63 \% \text { men and } 59 \% \\
\text { women; }\end{array}$ \\
\hline
\end{tabular}




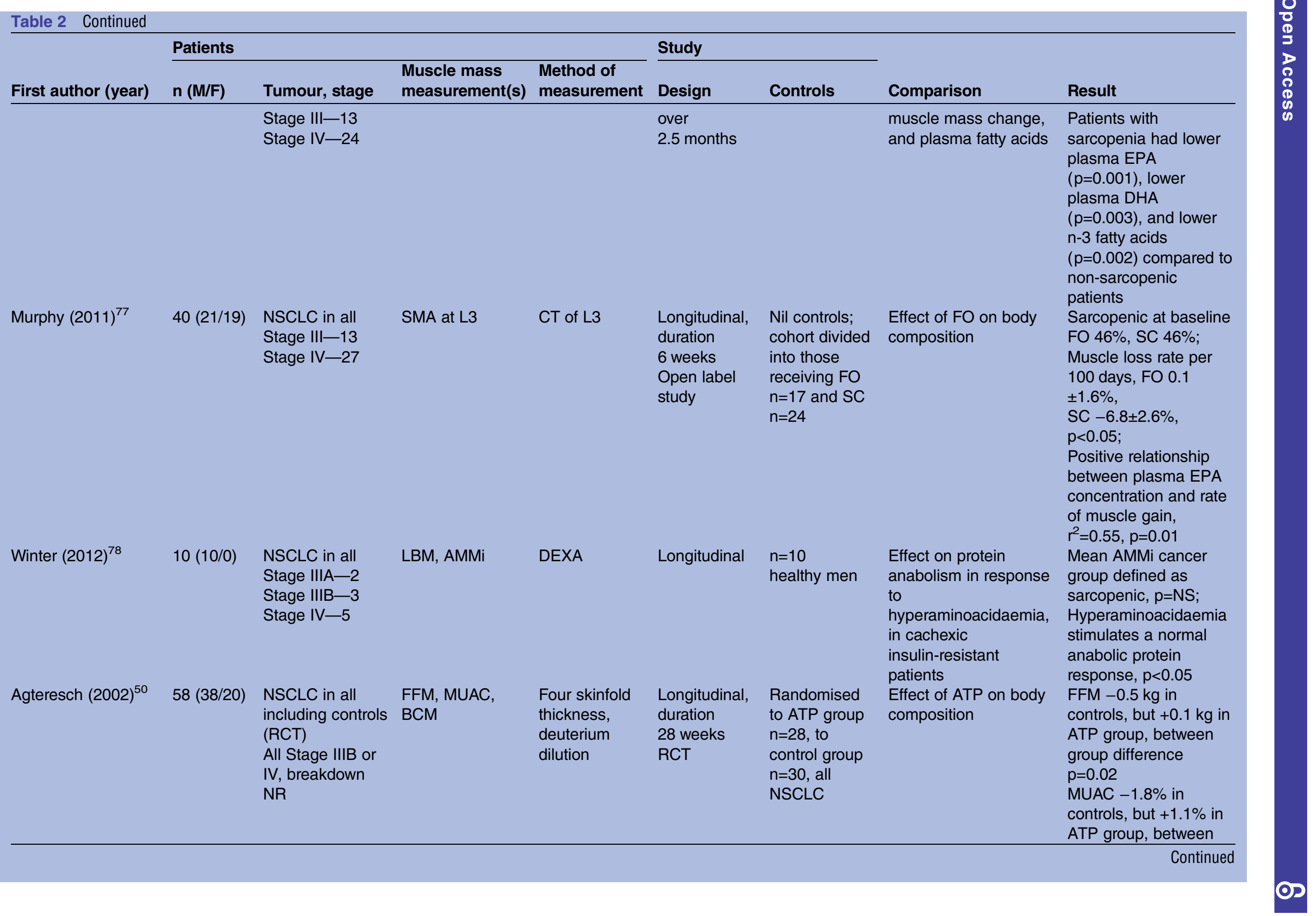




\begin{tabular}{|c|c|c|c|c|c|c|c|c|}
\hline \multirow[b]{2}{*}{ First author (year) } & \multicolumn{4}{|l|}{ Patients } & \multicolumn{2}{|l|}{ Study } & \multirow[b]{2}{*}{ Comparison } & \multirow[b]{2}{*}{ Result } \\
\hline & n (M/F) & Tumour, stage & $\begin{array}{l}\text { Muscle mass } \\
\text { measurement(s) }\end{array}$ & $\begin{array}{l}\text { Method of } \\
\text { measurement }\end{array}$ & Design & Controls & & \\
\hline & & & & & & & & $\begin{array}{l}\text { group difference } \\
p=0.02 \\
\text { BCM }-0.6 \% \text { per } \\
4 \text { weeks in controls, } \\
\text { but }-0.1 \% \text { in ATP } \\
\text { group, between group } \\
\text { diff } p=0.054\end{array}$ \\
\hline Beijer (2009) ${ }^{79}$ & $\begin{array}{l}n=100, \\
\text { with LC } \\
n=44 \\
n=57 \\
\text { completed } \\
\text { 8-week } \\
\text { study } \\
\text { period }\end{array}$ & $\begin{array}{l}\text { LC in } 44 \% \text { (most } \\
\text { frequent), colon } \\
\text { cancer } 13 \% \text {, } \\
\text { various other } \\
\text { cancers } 43 \% \\
\text { Stage NR } \\
\text { 'preterminal' }\end{array}$ & MUAC & $\begin{array}{l}\text { Upper arm } \\
\text { measurements }\end{array}$ & $\begin{array}{l}\text { Longitudinal, } \\
\text { duration } \\
8 \text { weeks } \\
\text { RCT }\end{array}$ & $\begin{array}{l}\text { Baseline: ATP } \\
n=51, \text { SC } \\
n=49 ; \\
\text { Completed } \\
\text { study: } \\
\text { ATP } n=29, \text { SC } \\
n=28\end{array}$ & $\begin{array}{l}\text { Effect of ATP on } \\
\text { nutritional status and } \\
\text { survival }\end{array}$ & $\begin{array}{l}\text { Post ATP loss of } \\
\text { MUAC } \\
-2.24 \mathrm{~mm}, \mathrm{SC} \text { group } \\
-1.52 \mathrm{~mm}, \mathrm{p}=\mathrm{NS} \\
\text { Short term } 0-8 \text { weeks } \\
\text { survival benefit with } \\
\text { ATP (HR } 0.17 \text {, } \\
\mathrm{p}=0.023), \text { and long } \\
\text { term } 0-6 \text { months } \\
\text { survival benefit (HR } \\
0.35, p=0.025)\end{array}$ \\
\hline
\end{tabular}

ALM, appendicular lean mass; AMA, arm muscle area; AMMi, appendicular muscle mass index; BCM, body cell mass; BCMi, BCM index; BFMAMA, bone free mid arm muscle area; BIA, bioelectrical impedance analysis; BMI, body mass index; COPD, chronic obstructive pulmonary disease; CRP, C reactive protein; CT of L3, CT of the third lumbar space; DEXA, dual-energy X-ray absorptiometry; DL, detectable leptin; EPA, eicosapentaenoic acid; F, female; FFM, fat-free mass; FFMi, FFM index; FO, fish oil; GCR, gastric and colorectal cancer; GI, gastrointestinal; HV, healthy volunteers; IL-15, interleukin 15; ILGF, insulin-like growth factor; LBM, lean body mass; LC, lung cancer; M, male; MI, muscle index; MUAC, mid-upper arm circumference; MA, muscle area; NR, not recorded; NS, non-significant; NSCLC, non-small cell lung cancer; PS, performance status; RCT, randomised controlled trial; REE, resting energy expenditure; SCLC, small cell lung cancer; SMA at L3 or T4, skeletal muscle area at the level of the lumbar vertebra L3 or thoracic vertebra T4; SMAi, skeletal muscle area index; SMMI, skeletal muscle mass index; TNM, tumour, node, metastasis; HCC, hepatocellular carcinoma; MAMC, mid-arm circumference; PIF, proteolysis inducing factor. 


\begin{tabular}{|c|c|c|c|c|c|c|c|c|}
\hline \multirow[b]{2}{*}{ First author (year) } & \multicolumn{4}{|l|}{ Patients } & \multicolumn{2}{|l|}{ Study } & \multirow[b]{2}{*}{ Comparison } & \multirow[b]{2}{*}{ Result } \\
\hline & $N(M / F)$ & Tumour, stage & $\begin{array}{l}\text { Muscle function } \\
\text { and muscle mass } \\
\text { measurements }\end{array}$ & $\begin{array}{l}\text { Method of } \\
\text { measurement }\end{array}$ & Design & Controls & & \\
\hline Jagoe $(2001)^{27}$ & $60(43 / 17)$ & LC in all & $\begin{array}{l}\text { Grip strength } \\
\text { Z-score } \\
\text { FFM, MAMC, } \\
\text { BFMAMA }\end{array}$ & $\begin{array}{l}\text { HDA dynamometer } \\
\text { BIA, four } \\
\text { skinfold-thickness, } \\
\text { upper arm } \\
\text { measurements }\end{array}$ & $\begin{array}{l}\text { Cross } \\
\text { sectional }\end{array}$ & $\begin{array}{l}\mathrm{n}=22 \text {, mild } \\
\text { COPD }\end{array}$ & $\begin{array}{l}\text { Nutritional status } \\
\text { of patients } \\
\text { undergoing lung } \\
\text { cancer } \\
\text { operations }\end{array}$ & $\begin{array}{l}\text { Grip strength in } \\
\text { absolute terms or } \\
\text { Z-score no } \\
\text { difference LC vs } \\
\text { controls, } p=N S \\
\text { No difference in } \\
\text { FFMi and BFMAMA } \\
\text { comparing LC and } \\
\text { controls, all } p=N S\end{array}$ \\
\hline Fearon $(2006)^{28}$ & $\begin{array}{l}518 \\
(355 / 163)\end{array}$ & $\begin{array}{l}\text { LC } n=231 \\
\text { Upper GI cancer } \\
n=198 \\
\text { Other GI cancer } \\
n=89 \\
\text { Stage NR }\end{array}$ & LBM & $\mathrm{BIA}$ & $\begin{array}{l}\text { RCT (double } \\
\text { blind, placebo } \\
\text { controlled, } \\
\text { randomised) }\end{array}$ & Nil & $\begin{array}{l}\text { Effect of } 2 \mathrm{~g} \text { and } \\
4 \mathrm{~g} \text { doses of EPA } \\
\text { diester vs } \\
\text { placebo in the } \\
\text { process of } \\
\text { cachexia }\end{array}$ & $\begin{array}{l}\text { Patient-reported } \\
\text { physical functioning } \\
\text { increased by } 7 \% \text { in } \\
\text { group receiving } 2 \mathrm{~g} \\
\text { EPA compared with } \\
\text { controls ( } p=0.04)\end{array}$ \\
\hline Tozer $(2008)^{54}$ & $\begin{array}{l}66(49 / 17) \\
\text { only } 35 \\
\text { completed } \\
\text { study }\end{array}$ & $\begin{array}{l}\text { All LC } \\
\text { Stage NR }\end{array}$ & BCM & NR & $\begin{array}{l}\text { RCT (double } \\
\text { blind, placebo } \\
\text { controlled, } \\
\text { randomised) }\end{array}$ & Nil & $\begin{array}{l}\text { Effect of } \\
\text { cysteine-rich } \\
\text { protein } \\
\text { supplement on } \\
\text { body weight and } \\
\text { body cell mass }\end{array}$ & $\begin{array}{l}\text { Handgrip force } \\
\text { improved by }+12.41 \\
\pm 16.52 \% \text { in cysteine } \\
\text { group compared to } \\
\text { baseline }(p=0.019)\end{array}$ \\
\hline Trutschnigg (2008) ${ }^{17}$ & $\begin{array}{l}81(\mathrm{NR} / \\
\mathrm{NR}) \\
74 \\
\text { completed } \\
\text { muscle } \\
\text { function } \\
\text { tests } \\
(48 / 26)\end{array}$ & $\begin{array}{l}\text { Patients with } \\
\text { advanced } \\
\text { NSCLC and } \\
\text { gastrointestinal } \\
\text { cancer, } \\
\text { breakdown NR } \\
\text { Stage NR }\end{array}$ & $\begin{array}{l}\text { Handgrip strength } \\
\text { In Newton metre } \\
\text { for Biodex, and } \\
\text { pounds for Jamar } \\
\text { FFM }\end{array}$ & $\begin{array}{l}\text { Jamar and Biodex } \\
\text { dynamometer } \\
\text { ( } n=74 \text { completed) } \\
\text { DEXA, BIA ( } n=70 \\
\text { completed) }\end{array}$ & $\begin{array}{l}\text { Cross } \\
\text { sectional }\end{array}$ & Nil & $\begin{array}{l}\text { Relationship } \\
\text { between DEXA } \\
\text { and BIA, and } \\
\text { Jamar and } \\
\text { Biodex } \\
\text { dynamometry } \\
\text { and their } \\
\text { precision in } \\
\text { patients with } \\
\text { advanced cancer }\end{array}$ & $\begin{array}{l}\text { Biodex HGS mean } \\
\pm \text { SD: men } 47.8 \\
\pm 13.6 \text { vs women } \\
32.7 \pm 9.3, p<0.05 \\
\text { Jamar HGS mean } \\
\pm \text { SD: } \\
\text { men } 78.5 \pm 21.6 \text { vs } \\
\text { women } 49.7 \pm 13.5 \text {, } \\
\text { p<0.001; } \\
\% C V \text { biodex } 16.7 \% \text {, } \\
\text { Jamar } 6.3 \% \\
\text { Wide limits of } \\
\text { agreement in } \\
\text { determining FFM, } \\
\text { DEXA v BIA, } \\
\text { p=NS, but low \%CV } \\
\text { for FFM DEXA }\end{array}$ \\
\hline
\end{tabular}




\begin{tabular}{|c|c|c|c|c|c|c|c|c|}
\hline \multirow[b]{2}{*}{ First author (year) } & \multicolumn{4}{|l|}{ Patients } & \multicolumn{2}{|l|}{ Study } & \multirow[b]{2}{*}{ Comparison } & \multirow[b]{2}{*}{ Result } \\
\hline & $\mathrm{N}(\mathrm{M} / \mathrm{F})$ & Tumour, stage & $\begin{array}{l}\text { Muscle function } \\
\text { and muscle mass } \\
\text { measurements }\end{array}$ & $\begin{array}{l}\text { Method of } \\
\text { measurement }\end{array}$ & Design & Controls & & \\
\hline & & & & & & & & $\begin{array}{l}(0.79) \text { and } B I A \\
(0.42)\end{array}$ \\
\hline Kilgour $(2010)^{53}$ & $\begin{array}{l}N=84, \text { with } \\
L C 16 \\
(19 \%) \text { of } \\
\text { cohort } \\
(48 / 36)\end{array}$ & $\begin{array}{l}\text { Metastatic } 57 \% \text {, } \\
\text { locally advanced } \\
43 \% \text {, stage NR }\end{array}$ & $\begin{array}{l}\text { HGS in kg, QS in } \\
\text { Newton metre } \\
\text { SMMI, ALM }\end{array}$ & $\begin{array}{l}\text { Jamar (HGS) and } \\
\text { Biodex (QS) } \\
\text { DEXA }\end{array}$ & $\begin{array}{l}\text { Cross } \\
\text { sectional }\end{array}$ & Nil & $\begin{array}{l}\text { Relationship of } \\
\text { fatigue to muscle } \\
\text { mass and } \\
\text { strength }\end{array}$ & $\begin{array}{l}\text { HGS on fatigue, } \\
95 \% \mathrm{Cl}-1.1 \text { to } \\
-0.15, p<0.05 ; \\
\mathrm{QS} \text { on fatigue, } 95 \% \\
\mathrm{Cl}-0.2 \text { to }-0.01, \\
\mathrm{p}<0.05 \text {; } \\
\text { Brief fatigue index } \\
\text { associated with } \\
\text { SMMI }(95 \% \mathrm{Cl}-8.4 \\
\text { to }-1.3) \mathrm{p}<0.01 \text {, and } \\
\text { sarcopenia, } p<0.01\end{array}$ \\
\hline Vigano $(2009)^{52}$ & $\begin{array}{l}N=172 \\
(101 / 71)\end{array}$ & $\begin{array}{l}\text { NSCLC } n=64 \text {, } \\
\text { Stage III and IV, } \\
\text { breakdown NR } \\
\text { Metastatic GI } \\
\text { cancer } n=108\end{array}$ & $\begin{array}{l}\text { Handgrip force } \\
\text { and percentile } \\
\text { LBM, ALM }\end{array}$ & $\begin{array}{l}\text { Jamar } \\
\text { dynamometer } \\
\text { DEXA }(n=64)\end{array}$ & $\begin{array}{l}\text { Cross } \\
\text { sectional }\end{array}$ & Nil & $\begin{array}{l}\text { ACE gene } \\
\text { polymorphism } \\
\text { (insertion }{ }^{2}-\text { II, } \\
\text { insertion/ } \\
\text { deletion-ID, } \\
\text { deletion }{ }^{2}-D D \text { ) on } \\
\text { nutritional status }\end{array}$ & $\begin{array}{l}\text { DD allele group } \\
\text { showed greater } \\
\text { handgrip force and } \\
\text { grip percentile than } \\
\text { II group, } p<0.05 \text {; but } \\
\text { no difference in LBM } \\
\text { or ALM } p=N S \\
\text { Trend ( } p=0.07 \text { ) } \\
\text { towards lower LBM } \\
\text { in ID compared to II } \\
\text { groups }\end{array}$ \\
\hline $\begin{array}{l}\text { Peddle-McIntyre } \\
(2012)^{56}\end{array}$ & $17(7 / 10)$ & $\begin{array}{l}\text { NSCLC } n=16 \\
\text { Stage I-II-11 } \\
\text { Stage III-5 } \\
\text { Limited stage } \\
\text { SCLC } n=1\end{array}$ & $\begin{array}{l}\text { Chest press, Leg } \\
\text { press, functional } \\
\text { performance } \\
\text { measure (6MWD } \\
\text {-6-min walk } \\
\text { distance, GUAG, } \\
\text { chair stands and } \\
\text { arm curls in } 30 \mathrm{~s} \text { ) } \\
\text { LBM, ALM }\end{array}$ & $\begin{array}{l}\text { 1RM in } \mathrm{kg} \\
\text { DEXA }\end{array}$ & $\begin{array}{l}\text { Longitudinal, } \\
\text { duration } \\
10 \text { weeks }\end{array}$ & Nil & $\begin{array}{l}\text { Resistance } \\
\text { exercise training } \\
\text { efficacy and } \\
\text { feasibility in lung } \\
\text { cancer survivors }\end{array}$ & $\begin{array}{l}\text { Mean change from } \\
\text { baseline to end of } \\
\text { training in } 95 \% \mathrm{Cl} \\
\text { chest press } 12.3 \text { to } \\
17.5 \text {, leg press } 23.5 \\
\text { to } 39.8,6 \mathrm{MWD} 48 \\
\text { to } 124, \text { GUAG }-0.4 \\
\text { to }-1.2 \text {, chair stands } \\
2.3 \text { to } 6.1 \text {, arm curls } \\
2.1 \text { to } 5.1 \text {, all p<0.05 } \\
\text { LBM and ALM no } \\
\text { change from }\end{array}$ \\
\hline
\end{tabular}




\begin{tabular}{|c|c|c|c|c|c|c|c|c|}
\hline \multirow[b]{2}{*}{ First author (year) } & \multicolumn{4}{|l|}{ Patients } & \multicolumn{2}{|l|}{ Study } & \multirow[b]{2}{*}{ Comparison } & \multirow[b]{2}{*}{ Result } \\
\hline & $N(M / F)$ & Tumour, stage & $\begin{array}{l}\text { Muscle function } \\
\text { and muscle mass } \\
\text { measurements }\end{array}$ & $\begin{array}{l}\text { Method of } \\
\text { measurement }\end{array}$ & Design & Controls & & \\
\hline $\begin{array}{l}\text { Martinez-Hernandez } \\
(2012)^{61}\end{array}$ & $21(19 / 2)$ & $\begin{array}{l}\text { Lung cancer } \\
n=13 \text {, Gl cancer } \\
n=6, \text { Other } \\
\text { cancer } n=2 \\
\text { Stage according } \\
\text { to tumour group } \\
\text { NR }\end{array}$ & $\begin{array}{l}\text { HGS and treadmill } \\
\text { GMWT } \\
\text { FFM }\end{array}$ & BIA & Longitudinal & $\begin{array}{l}\mathrm{n}=8 \\
\text { healthy } \\
\text { volunteers }\end{array}$ & $\begin{array}{l}\text { The role of IL-15 } \\
\text { in patients with } \\
\text { cachectic cancer }\end{array}$ & $\begin{array}{l}\text { baseline to post } \\
\text { training, all } \mathrm{p}=\mathrm{NS} \\
\text { HGS no difference } \\
\text { comparing cachectic } \\
\text { group to controls, } \\
\mathrm{p}=\mathrm{NS} ; \\
6 \mathrm{MWT} \text { in cachectic } \\
\text { group } 369 \pm 73 \mathrm{~m} \text { vs } \\
474 \pm 57 \mathrm{~m}, \mathrm{p}<0.05\end{array}$ \\
\hline $\begin{array}{l}\text { Op den Kamp } \\
(2012)^{55}\end{array}$ & $16(15 / 1)$ & $\begin{array}{l}\text { NSCLC in all } \\
\text { Stage I-II-11 } \\
\text { Stage IIIA-2 } \\
\text { Stage IIIB-3 }\end{array}$ & $\begin{array}{l}\text { Intensity of } \\
\text { physical activity } \\
\text { FFMi }\end{array}$ & $\begin{array}{l}\text { Triaxial } \\
\text { accelerometer } \\
\text { (Tracmor) in } \\
\text { counts/min } \\
\text { DEXA }\end{array}$ & $\begin{array}{l}\text { Cross } \\
\text { sectional }\end{array}$ & $\begin{array}{l}n=10 \\
\text { healthy } \\
\text { volunteers }\end{array}$ & $\begin{array}{l}\text { Skeletal muscle } \\
\text { ubiquitin } \\
\text { proteasome } \\
\text { system activity in } \\
\text { precachexia }\end{array}$ & $\begin{array}{l}\text { High intensity } \\
\text { physical activity in } \\
\text { LC vs controls } \\
p=0.049 ; \\
\text { FFMi no significant } \\
\text { difference in } \\
\text { precachectic cancer } \\
\text { vs controls, } p=N S\end{array}$ \\
\hline $\begin{array}{l}\text { Op den Kamp } \\
(2013)^{62}\end{array}$ & $26(17 / 9)$ & $\begin{array}{l}\text { NSCLC } \\
\text { Stage IIIB-10 } \\
\text { Stage IV-16 }\end{array}$ & $\begin{array}{l}\text { QS) } \\
\text { FFMi, AMMi }\end{array}$ & DEXA & $\begin{array}{l}\text { Cross } \\
\text { sectional }\end{array}$ & $\begin{array}{l}n=10 \\
\text { healthy } \\
\text { volunteers }\end{array}$ & $\begin{array}{l}\text { Expression of } \\
\text { signalling } \\
\text { molecules in } \\
\text { protein } \\
\text { metabolism in } \\
\text { lung cancer } \\
\text { cachexia }\end{array}$ & $\begin{array}{l}\text { QS } 31 \% \text { lower in } \\
\text { cachectic group } \\
\text { compared to } \\
\text { controls, } p<0.05\end{array}$ \\
\hline
\end{tabular}




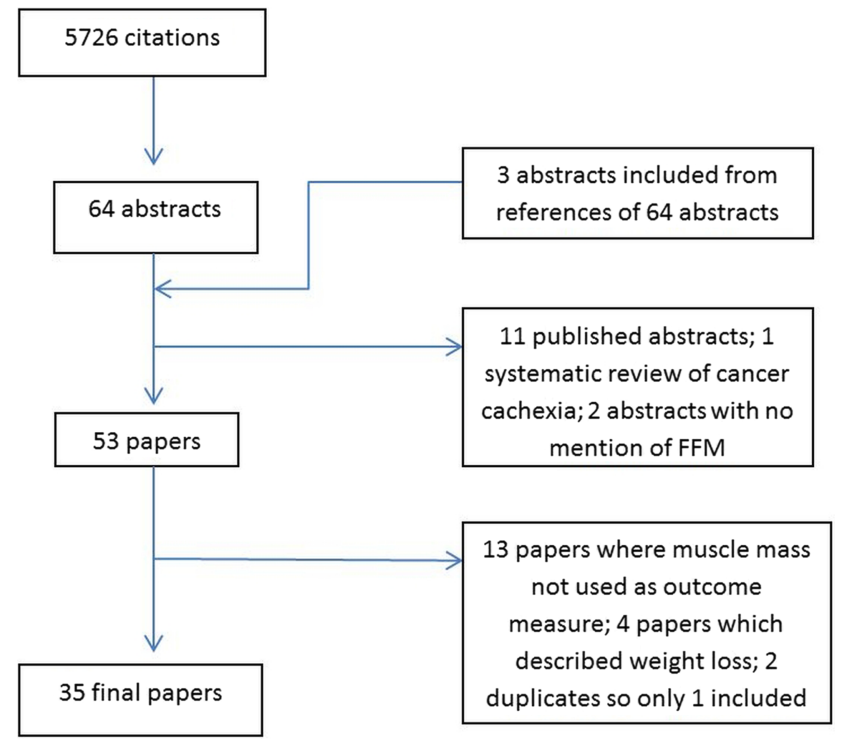

Figure 1 Flowchart showing a breakdown of the results of our search using the search terms used and the derivation of articles for final analysis. FFM, fat-free mass.

proteolytic pathway may not be activated. ${ }^{55}{ }^{80}$ Different ACE-gene polymorphism allelic combinations ${ }^{52}$ and leptin expression ${ }^{70}$ have not been shown to have significant effects on muscle mass. FFM is the major determinant of energy metabolism in humans, represented by resting energy expenditure (REE), and there exists a linear relationship between REE and FFM in healthy adults. ${ }^{81}$ In lung cancer cachexia, this relationship seems to be distorted ${ }^{69}{ }^{71}$ but results have been conflicting as to whether REE contributes to the development of lung cancer cachexia. ${ }^{67687273}$

The use of CT images for diagnosis of muscle mass depletion alone showed a high prevalence of this phenomenon in patients with NSCLC, ${ }^{25}$ regardless of body mass index and even among the obese. ${ }^{1}$ CT images were also used to chart progressive muscle loss over time, and to create a prognostic model for survival based on weight loss, muscle mass and muscle attenuation. ${ }^{65} 66$ The presence of muscle mass attenuation was associated with poorer functional status and overall survival.

Nine interventional studies explored the effect of either nutritional supplements or ATP infusions on muscle mass and function. A randomised controlled study with 518 participants, examining the effect of eicosapentaenoic acid (found in fish oil) supplements, revealed increased patient-rated physical functioning, but no significant change in FFM, at the end of the study period. ${ }^{28} \mathrm{~A}$ similar, smaller study of eight participants concurred ${ }^{75}$ By contrast, one study of fish oil supplementation demonstrated a reduction in the rate of loss of muscle mass; however, the number of studies was small-40 patients in total. In addition, those considered sarcopenic were found to have lower plasma fatty acids than those without, in a study with 41 patients with NSCLC. ${ }^{76} \quad 77$ An interventional randomised controlled study with 66 participants found that cysteine-rich protein supplements increased FFM, as well as HGS, compared with conventional protein supplements, ${ }^{54}$ and a small case-control study with 10 patients found that hyperaminoacidaemia stimulated a normal anabolic protein response even in the presence of insulin resistance, in patients with cancer cachexia. ${ }^{78}$ Two randomised controlled studies investigating the effect of ATP infusions on body composition gave conflicting reports, one $(\mathrm{N}=58)$ finding that ATP slowed the rate of loss of muscle mass ${ }^{50}$ while the other $(\mathrm{N}=100)$ did not. ${ }^{79}$ Only the study by Fearon $e t a t^{28}$ described power calculations to detect a statistically significant difference.

\section{Degree of sarcopenia or loss of muscle mass and physical functioning}

There was very little direct evaluation of the relationship between muscle mass and muscle function. However, the studies that evaluated muscle mass alongside muscle function show that there is limited correlation. Muscle strength seemed to be affected, regardless of loss of muscle mass. In patients with precachexia, exercise capacity was significantly reduced, despite maintenance of muscle mass ${ }^{55}$ and resistance exercise training increased all parameters of muscle strength and physical performance, with no difference to muscle mass. ${ }^{56}$ In this review, patients with cachexia showed reduced strength in terms of walking distance ${ }^{61}$ and quadriceps strength $^{62}$ compared with controls.

\section{DISCUSSION}

Loss of muscle mass, as part of a weight-losing syndrome, is a central feature of cancer cachexia. However, changes in muscle mass, and/or performance, may predate clinically overt cachexia, as part of ageing or secondary to inflammation/disuse. This implies that consideration of both muscle mass and function, rather than weight loss alone, is clinically important. Understanding this relationship, and the factors associated with each, will provide opportunities for focused intervention to improve clinical outcomes.

The findings of our review highlight several important issues. While studies exploring molecular and metabolic factors associated with loss of muscle mass have contributed to a better understanding of the pathophysiology of cancer cachexia, there remains considerable uncertainty in relation to mechanisms. Our review in patients with lung cancer demonstrates inconsistency of findings as to the factors implicated in the development of cachexia, compared with other cancer sites. It highlights the lack of clear therapeutic targets and emphasises the need for concerted, appropriately-sized exploration of predictive and prognostic factors in lung cancer cachexia.

This uncertainty with regard to precise pathophysiological mechanisms is reflected in the lack of consistent effect of interventions aimed at slowing the rate of loss 
of muscle mass and improving muscle function in lung cancer. Studies reviewed which refer to cachexia management support a multimodal approach, including targeted exercise, nutritional counselling, social support and pharmacological intervention. ${ }^{82}$ This review highlights inherent challenges of such an approach, with nutritional interventions in particular failing to demonstrate efficacy, ${ }^{83}$ although the role of exercise is emerging. ${ }^{56}{ }^{84}$ It also suggests the need to represent patients with NSCLC adequately within trials of new interventions, such as myostatin antibody therapies, rather than assuming a class effect across tumour sites.

Strikingly, our review has demonstrated that, to date, there has not been due attention to the concept of sarcopenia as a distinct, if overlapping syndrome, in a condition affecting a largely elderly population. It also highlights the difficulty in making comparisons between studies, due to the heterogeneity of methods employed in measuring muscle mass and different cut-off values used for defining sarcopenia. Current standardised values were derived from a large elderly cohort and the cut-off values were based on healthy young adult reference values. These values have been used to define sarcopenia in cancer, ${ }^{13}{ }^{85}$ including one in this review. ${ }^{53}$ The relevance of this definition to patients with cancer is debatable, for a number of reasons. First, sarcopenia manifests in patients with cancer of all ages and is not isolated to the elderly alone. Second, the pathophysiology of sarcopenia in patients with cancer may differ at least in part to that of the non-cancer elderly population. ${ }^{86}$ With this in mind, the more recent international consensus document recommending a reference value of absolute muscularity below the fifth centile is to be welcomed. ${ }^{13}$ Finally, the recognition of muscle strength and performance as a defining component of sarcopenia in the elderly patients needs consideration, within the context of cancer cachexia.

The argument for the objective evaluation of physical performance is pertinent, particularly as part of the definitive assessment of sarcopenia alongside measurements of muscle mass. Currently, physical fitness for treatment is determined largely by the PS score. This score is imperfect as it is subjective, with reports of interobserver variability, ${ }^{87}$ and there is only a modest correlation between PS and observed physical performance. ${ }^{88}$ Interclinician PS discordance has led to a lower percentage of PS 0 and 1 patients appearing to get chemotherapy, ${ }^{89}$ and has led to a call for objective evaluation of physical functioning. ${ }^{90}$ Some proposed methods include tests of gait speed and muscle strength. It is postulated that objective measures of muscle mass and strength together may complement, or even outperform, PS as a predictor of fitness for systemic treatment, provided that they can be readily performed in routine clinical settings.

Our review has several limitations. The heterogeneity of the studies included in this review made it difficult to account for individual risk of bias, not least because we included a broad range of studies from large randomised controlled trials to small observational studies. This limitation also means that some articles included in this review, while being relevant to sarcopenia, were more broadly related to muscle mass outcomes in cancer cachexia, rather than assessing sarcopenia directly. Our search also was limited to studies published in English, and although our review included some studies with negative or inconclusive findings, there may indeed exist some publication bias for which we are unable to account

The article by Temel et $a l,{ }^{91}$ which demonstrated that early palliative care involvement increased patient survival, as well as quality of life, has highlighted the importance of supportive measures in a poor-prognosis population receiving active oncological intervention. As such, focusing research on the identification and management of sarcopenia in patients with lung cancer may prove to be a tolerable and cost-effective adjunct to the current lung cancer care.

While development of a clearer definition of cancer cachexia provides an additional component of a robust, objective clinical framework for stratification of patients for focused interventions, the enhanced role of muscle strength/performance as a defining assessment of sarcopenia requires attention. A standardised definition of cancer-related sarcopenia which can be used clinically and in the research setting will harmonise reporting, allowing for direct comparison of results as well as meta-analysis of data. In the era of stratified medicine, this review identifies opportunities to examine cellular and genetic factors associated with sarcopenia in lung cancer coherently and to link them with changes in tumour phenotype which impact on morbidity and survival.

Contributors JCo and SN were responsible for the conception and design of this review, independently reviewed the citations, and were responsible for analysing and interpreting the data. JCo and BC conducted the searches. SN, $\mathrm{JCh}, \mathrm{AB}$ and $\mathrm{JCo}$ drafted the article and revised its content to its final version.

Funding $\mathrm{JCO}$ is funded by Cardiff and Vale University Health Board under the Clinical Research Fellowship scheme.

Competing interests None.

Provenance and peer review Not commissioned; externally peer reviewed.

Data sharing statement No additional data are available.

Open Access This is an Open Access article distributed in accordance with the Creative Commons Attribution Non Commercial (CC BY-NC 3.0) license, which permits others to distribute, remix, adapt, build upon this work noncommercially, and license their derivative works on different terms, provided the original work is properly cited and the use is non-commercial. See: http:// creativecommons.org/licenses/by-nc/3.0/

\section{REFERENCES}

1. Prado CM, Lieffers JR, McCargar LJ, et al. Prevalence and clinical implications of sarcopenic obesity in patients with solid tumours of the respiratory and gastrointestinal tracts: a population-based study. Lancet Oncol 2008;9:629-35.

2. Prado CM, Baracos VE, McCargar LJ, et al. Sarcopenia as a determinant of chemotherapy toxicity and time to tumor progression 
in metastatic breast cancer patients receiving capecitabine treatment. Clin Cancer Res 2009;15:2920-6.

3. Awad $\mathrm{S}$, Tan $\mathrm{BH}$, Cui $\mathrm{H}$, et al. Marked changes in body composition following neoadjuvant chemotherapy for oesophagogastric cancer. Clin Nutr 2012;31:74-7.

4. Mir O, Coriat R, Blanchet B, et al. Sarcopenia predicts early dose-limiting toxicities and pharmacokinetics of sorafenib in patients with hepatocellular carcinoma. PLoS ONE 2012;7:e37563.

5. Lieffers JR, Bathe OF, Fassbender K, et al. Sarcopenia is associated with postoperative infection and delayed recovery from colorectal cancer resection surgery. Br J Cancer 2012;107:931-6.

6. Cruz-Jentoft AJ, Baeyens JP, Bauer JM, et al. Sarcopenia: European consensus on definition and diagnosis: report of the European Working Group on sarcopenia in older people. Age Ageing 2010;39:412-23.

7. Janssen I, Heymsfield SB, Ross R. Low relative skeletal muscle mass (sarcopenia) in older persons is associated with functional impairment and physical disability. J Am Geriatr Soc 2002;50:889-96.

8. Baumgartner RN, Koehler KM, Gallagher D, et al. Epidemiology of sarcopenia among the elderly in New Mexico. Am J Epidemiol 1998;147:755-63.

9. Landi F, Liperoti R, Fusco D, et al. Sarcopenia and mortality among older nursing home residents. J Am Med Dir Assoc 2012;13:121-6.

10. Cherin P, Voronska E, Fraoucene N, et al. Prevalence of sarcopenia among healthy ambulatory subjects: the sarcopenia begins from 45 years. Aging Clin Exp Res 2013. 17 Oct 2013. Epub ahead of print.

11. Veasey-Rodrigues $\mathrm{H}$, Parsons HA, Janku F, et al. A pilot study of temsirolimus and body composition. J Cachexia Sarcopenia Muscle 2013;4:259-65.

12. Harimoto N, Shirabe K, Yamashita YI, et al. Sarcopenia as a predictor of prognosis in patients following hepatectomy for hepatocellular carcinoma. Br J Surg 2013;100:1523-30.

13. Fearon K, Strasser F, Anker SD, et al. Definition and classification of cancer cachexia: an international consensus. Lancet Oncol 2011;12:489-95.

14. Janssen I, Baumgartner RN, Ross R, et al. Skeletal muscle cut points associated with elevated physical disability risk in older men and women. Am J Epidemiol 2004;159:413-21.

15. Goodpaster BH, Park SW, Harris TB, et al. The loss of skeletal muscle strength, mass, and quality in older adults: the health, aging and body composition study. J Gerontol Ser A Biol Sci Med Sci 2006;61:1059-64.

16. Kendler DL, Borges JL, Fielding RA, et al. The official positions of the International Society for Clinical Densitometry: indications of use and reporting of DXA for body composition. J Clin Densitom 2013;16:496-507

17. Trutschnigg B, Kilgour RD, Reinglas J, et al. Precision and reliability of strength (Jamar vs. Biodex handgrip) and body composition (dual-energy X-ray absorptiometry vs. bioimpedance analysis) measurements in advanced cancer patients. Appl Physiol Nutr Metab 2008;33:1232-9.

18. Landi F, Russo A, Liperoti $R$, et al. Mid arm muscle circumference, physical performance and mortality: results from the aging and longevity study in the Sirente geographic area (ilSIRENTE study). Clin Nutr 2010;29:441-7.

19. Freiberger E, de Vreede P, Schoene D, et al. Performance-based physical function in older community-dwelling persons: a systematic review of instruments. Age Ageing 2012;41:712-21.

20. Greendale GA, DeAmicis TA, Bucur A, et al. A prospective study of the effect of fracture on measured physical performance: results from the MacArthur Study-MAC. J Am Geriatr Soc 2000;48: 546-9.

21. Ferlay J, Shin HR, Bray F, et al. Estimates of worldwide burden of cancer in 2008: GLOBOCAN 2008. Int $J$ Cancer 2010;127:2893-917.

22. Rachet B, Woods LM, Mitry E, et al. Cancer survival in England and Wales at the end of the 20th century. Br J Cancer 2008;99(Suppl 1) S2-10.

23. Vinod SK, Sidhom MA, Gabriel GS, et al. Why do some lung cancer patients receive no anticancer treatment? $J$ Thorac Oncol 2010;5:1025-32.

24. Pemberton L, Sumra P, Tetlow C, et al. Do treatment decisions made at lung cancer multi-disciplinary team meetings (MDTs) reflect the actual treatment given in practice? Lung Cancer 2013;79:S36.

25. Baracos VE, Reiman T, Mourtzakis M, et al. Body composition in patients with non-small cell lung cancer: a contemporary view of cancer cachexia with the use of computed tomography image analysis. Am J Clin Nutr 2010;91:1133S-7S.

26. Villasenor $\mathrm{A}$, Ballard-Barbash $\mathrm{R}$, Baumgartner $\mathrm{K}$, et al. Prevalence and prognostic effect of sarcopenia in breast cancer survivors: the HEAL Study. J Cancer Surviv 2012;6:398-406.
27. Jagoe RT, Goodship TH, Gibson GJ. Nutritional status of patients undergoing lung cancer operations. Ann Thorac Surg 2001;71:929-35.

28. Fearon KC, Barber MD, Moses AG, et al. Double-blind, placebocontrolled, randomized study of eicosapentaenoic acid diester in patients with cancer cachexia. J Clin Oncol 2006;24:3401-7.

29. Harvie MN, Campbell IT, Thatcher N, et al. Changes in body composition in men and women with advanced nonsmall cell lung cancer (NSCLC) undergoing chemotherapy. J Hum Nutr Diet 2003;16:323-6.

30. Sanchez-Lara K, Turcott JG, Juarez E, et al. Association of nutrition parameters including bioelectrical impedance and systemic inflammatory response with quality of life and prognosis in patients with advanced non-small-cell lung cancer: a prospective study. Nutr Cancer 2012;64:526-34.

31. Granger CL, McDonald CF, Parry SM, et al. Functional capacity, physical activity and muscle strength assessment of individuals with non-small cell lung cancer: a systematic review of instruments and their measurement properties. BMC Cancer 2013;13:135.

32. Blum D, Omlin A, Baracos VE, et al. Cancer cachexia: a systematic literature review of items and domains associated with involuntary weight loss in cancer. Crit Rev Oncol Hematol 2011;80:114-44.

33. Bruera $\mathrm{E}$, Ernst $\mathrm{S}$, Hagen $\mathrm{N}$, et al. Effectiveness of megestrol acetate in patients with advanced cancer: a randomized, double-blind, crossover study. Cancer Prev Control 1998;2:74-8.

34. Lindsey AM, Piper BF. Anorexia and weight loss: indicators of cachexia in small cell lung cancer. Nutr Cancer 1985;7:65-76.

35. Wolf RF, Pearlstone DB, Newman E, et al. Growth hormone and insulin reverse net whole body and skeletal muscle protein catabolism in cancer patients. Ann Surg 1992;216:280-90.

36. Gioulbasanis I, Baracos VE, Giannousi Z, et al. Baseline nutritional evaluation in metastatic lung cancer patients: mini nutritional assessment versus weight loss history. Ann Oncol 2011;22:835-41.

37. Jamieson NB, Brown DJ, Michael Wallace A, et al. Adiponectin and the systemic inflammatory response in weight-losing patients with non-small cell lung cancer. Cytokine 2004;27:90-2.

38. Melville S, McNurlan MA, Calder AG, et al. Increased protein turnover despite normal energy metabolism and responses to feeding in patients with lung cancer. Cancer Res 1990;50:1125-31.

39. Richards EW, Long CL, Nelson KM, et al. Protein turnover in advanced lung cancer patients. Metabolism 1993;42:291-6.

40. Staal-van den Brekel AJ, Schols AM, Dentener MA, et al. Metabolism in patients with small cell lung carcinoma compared with patients with non-small cell lung carcinoma and healthy controls. Thorax 1997;52:338-41.

41. Staal-van den Brekel AJ, Schols AM, ten Velde GP, et al. Analysis of the energy balance in lung cancer patients. Cancer Res 1994;54:6430-3.

42. van der Meij BS, Schoonbeek CP, Smit EF, et al. Pre-cachexia and cachexia at diagnosis of stage III non-small-cell lung carcinoma: an exploratory study comparing two consensus-based frameworks. $\mathrm{Br} \mathrm{J}$ Nutr 2013;109:2231-9.

43. Ovesen L, Hannibal J, Mortensen EL. The interrelationship of weight loss, dietary intake, and quality of life in ambulatory patients with cancer of the lung, breast, and ovary. Nutr Cancer 1993;19:159-67.

44. Richards EW, Long CL, Nelson KM, et al. Glucose metabolism in advanced lung cancer patients. Nutrition 1992;8:245-51.

45. Simons JP, Schols AM, Westerterp KR, et al. The use of bioelectrical impedance analysis to predict total body water in patients with cancer cachexia. Am J Clin Nutr 1995;61:741-5.

46. Arrieta $\mathrm{O}$, Michel Ortega RM, Villanueva-Rodriguez G, et al. Association of nutritional status and serum albumin levels with development of toxicity in patients with advanced non-small cell lung cancer treated with paclitaxel-cisplatin chemotherapy: a prospective study. BMC Cancer 2010;10:50.

47. Jatoi A, Ritter HL, Dueck A, et al. A placebo-controlled, double-blind trial of infliximab for cancer-associated weight loss in elderly and/or poor performance non-small cell lung cancer patients (N01C9). Lung Cancer 2010;68:234-9.

48. Meek CL, Wallace AM, Forrest LM, et al. The relationship between the insulin-like growth factor-1 axis, weight loss, an inflammation-based score and survival in patients with inoperable non-small cell lung cancer. Clin Nutr 2010;29:206-9.

49. Toso S, Piccoli A, Gusella M, et al. Altered tissue electric properties in lung cancer patients as detected by bioelectric impedance vector analysis. Nutrition 2000;16:120-4.

50. Agteresch HJ, Rietveld T, Kerkhofs LG, et al. Beneficial effects of adenosine triphosphate on nutritional status in advanced lung cancer patients: a randomized clinical trial. J Clin Oncol 2002;20:371-8.

51. Dagnelie PC, Agteresch HJ. Promising effects of adenosine triphosphate infusion on nutritional status and quality of life in 
advanced non-small-cell lung cancer: a randomized clinical trial. Drug Dev Res 2003;59:146-51.

52. Vigano A, Trutschnigg B, Kilgour RD, et al. Relationship between angiotensin-converting enzyme gene polymorphism and body composition, functional performance, and blood biomarkers in advanced cancer patients. Clin Cancer Res 2009;15:2442-7.

53. Kilgour RD, Vigano A, Trutschnigg B, et al. Cancer-related fatigue: the impact of skeletal muscle mass and strength in patients with advanced cancer. J Cachexia Sarcopenia Muscle 2010;1:177-85.

54. Tozer RG, Tai P, Falconer W, et al. Cysteine-rich protein reverses weight loss in lung cancer patients receiving chemotherapy or radiotherapy. Antioxid Redox Signal 2008;10:395-402.

55. Op den Kamp CM, Langen RC, Minnaard R, et al. Pre-cachexia in patients with stages I-III non-small cell lung cancer: systemic inflammation and functional impairment without activation of skeletal muscle ubiquitin proteasome system. Lung Cancer 2012;76:112-17.

56. Peddle-Mclntyre CJ, Bell G, Fenton D, et al. Feasibility and preliminary efficacy of progressive resistance exercise training in lung cancer survivors. Lung Cancer 2012;75:126-32.

57. McMillan DC, Watson WS, O'Gorman P, et al. Albumin concentrations are primarily determined by the body cell mass and the systemic inflammatory response in cancer patients with weight loss. Nutr Cancer 2001;39:210-13.

58. Crown AL, Cottle K, Lightman SL, et al. What is the role of the insulin-like growth factor system in the pathophysiology of cancer cachexia, and how is it regulated? Clin Endocrinol 2002;56:723-33.

59. Jagoe RT, Redfern CP, Roberts RG, et al. Skeletal muscle mRNA levels for cathepsin $\mathrm{B}$, but not components of the ubiquitinproteasome pathway, are increased in patients with lung cancer referred for thoracotomy. Clin Sci 2002;102:353-61.

60. Wieland BM, Stewart GD, Skipworth RJ, et al. Is there a human homologue to the murine proteolysis-inducing factor? Clin Cancer Res 2007:13:4984-92.

61. Martinez-Hernandez PL, Hernanz-Macias A, Gomez-Candela C, et al. Serum interleukin-15 levels in cancer patients with cachexia. Oncol Rep 2012;28:1443-52.

62. Op den Kamp CM, Langen RC, Snepvangers FJ, et al. Nuclear transcription factor kappa B activation and protein turnover adaptations in skeletal muscle of patients with progressive stages of lung cancer cachexia. Am J Clin Nutr 2013;98:738-48.

63. Harvie MN, Howell A, Thatcher N, et al. Energy balance in patients with advanced NSCLC, metastatic melanoma and metastatic breast cancer receiving chemotherapy-a longitudinal study. Br J Cancer 2005;92:673-80.

64. Bovio G, Bettaglio R, Bonetti G, et al. Evaluation of nutritional status and dietary intake in patients with advanced cancer on palliative care. Minerva Gastroenterol Dietol 2008:54:243-50.

65. Martin L, Birdsell L, Macdonald N, et al. Cancer cachexia in the age of obesity: skeletal muscle depletion is a powerful prognostic factor, independent of body mass index. J Clin Oncol 2013;31:1539-47.

66. Prado CM, Sawyer MB, Ghosh S, et al. Central tenet of cancer cachexia therapy: do patients with advanced cancer have exploitable anabolic potential? Am J Clin Nutr 2013;98:1012-19.

67. Hansell DT, Davies JWL, Burns HJG. The relationship between resting energy expenditure and weight loss in benign and malignant disease. Ann Surg 1986;203:240-5.

68. Fredix EWHM, Soeters PB, Wouters EFM, et al. Energy balance in relation to cancer cachexia. Clin Nutr 1990;9:319-24.

69. Staal-van den Brekel AJ, Schols AM, Dentener MA, et al. The effects of treatment with chemotherapy on energy metabolism and inflammatory mediators in small-cell lung carcinoma. $\mathrm{Br} J$ Cancer 1997;76:1630-5.

70. Simons JP, Schols AM, Campfield LA, et al. Plasma concentration of total leptin and human lung-cancer-associated cachexia. Clin $\mathrm{Sci}$ 1997;93:273-7.

71. Simons JP, Schols AM, Buurman WA, et al. Weight loss and low body cell mass in males with lung cancer: relationship with systemic inflammation, acute-phase response, resting energy expenditure, and catabolic and anabolic hormones. Clin Sci 1999;97:215-23.

72. Scott HR, McMillan DC, Watson WS, et al. Longitudinal study of resting energy expenditure, body cell mass and the inflammatory response in male patients with non-small cell lung cancer. Lung Cancer 2001;32:307-12.

73. Jatoi A, Daly BD, Hughes VA, et al. Do patients with nonmetastatic non-small cell lung cancer demonstrate altered resting energy expenditure? Ann Thorac Surg 2001;72:348-51.

74. Sarhill N, Mahmoud F, Walsh D, et al. Evaluation of nutritional status in advanced metastatic cancer. Support Care Cancer 2003;11:652-9.

75. Bauer JD, Capra S. Nutrition intervention improves outcomes in patients with cancer cachexia receiving chemotherapy-a pilot study. Support Care Cancer 2005;13:270-4.

76. Murphy RA, Mourtzakis M, Chu QS, et al. Skeletal muscle depletion is associated with reduced plasma ( $n-3)$ fatty acids in non-small cell lung cancer patients. $J$ Nutr 2010;140:1602-6.

77. Murphy RA, Mourtzakis M, Chu QS, et al. Nutritional intervention with fish oil provides a benefit over standard of care for weight and skeletal muscle mass in patients with nonsmall cell lung cancer receiving chemotherapy. Cancer 2011;117:1775-82.

78. Winter A, MacAdams J, Chevalier S. Normal protein anabolic response to hyperaminoacidemia in insulin-resistant patients with lung cancer cachexia. Clin Nutr 2012;31:765-73.

79. Beijer S, Hupperets PS, van den Borne BE, et al. Effect of adenosine $5^{\prime}$-triphosphate infusions on the nutritional status and survival of preterminal cancer patients. Anticancer Drugs 2009;20:625-33.

80. Khal J, Hine AV, Fearon KC, et al. Increased expression of proteasome subunits in skeletal muscle of cancer patients with weight loss. Int J Biochem Cell Biol 2005;37:2196-206.

81. Wang Z, Heshka S, Gallagher D, et al. Resting energy expenditure-fat-free mass relationship: new insights provided by body composition modeling. Am J Physiol Endocrinol Metab 2000;279:E539-45.

82. Vaughan VC, Martin P, Lewandowski PA. Cancer cachexia: impact, mechanisms and emerging treatments. J Cachexia Sarcopenia Muscle 2013;4:95-109.

83. Murphy RA, Yeung E, Mazurak VC, et al. Influence of eicosapentaenoic acid supplementation on lean body mass in cancer cachexia. Br J Cancer 2011;105:1469-73.

84. McClellan R. Exercise programs for patients with cancer improve physical functioning and quality of life. J Physiother 2013;59:57.

85. Boxer RS, Kenny AM, Dowsett R, et al. The effect of 6 months of androgen deprivation therapy on muscle and fat mass in older men with localized prostate cancer. Aging Male 2005;8:207-12.

86. Argiles JM, Busquets S, Felipe A, et al. Molecular mechanisms involved in muscle wasting in cancer and ageing: cachexia versus sarcopenia. Int J Biochem Cell Biol 2005;37:1084-104.

87. Sorensen JB, Klee M, Palshof T, et al. Performance status assessment in cancer patients. An inter-observer variability study. Br J Cancer 1993;67:773-5.

88. Montoya M, Fossella F, Palmer JL, et al. Objective evaluation of physical function in patients with advanced lung cancer: a preliminary report. J Palliat Med 2006:9:309-16.

89. May CH, Lester JF, Lee S. Performance status discordance and why it matters. Lung Cancer 2012;75(S1):S1-72.

90. Sonpavde G, Vogelzang NJ, Galsky MD, et al. Objective measures of physical functional capacity warrant exploration to complement or replace the subjective physician estimated performance status. Am J Clin Oncol 2012;35:163-6.

91. Temel JS, Greer JA, Muzikansky A, et al. Early palliative care for patients with metastatic non-small-cell lung cancer. $N$ Engl J Med 2010;363:733-42. 